\title{
Проявления процессов резонансного туннелирования и случайных флуктуаций потенциала с участием уровней квантовых точек в релаксации фототока $p-i-n$ GaAs/AlAs-гетероструктур
}

\author{
() Ю.Н. Ханин, Е.Е. Вдовин \\ Институт проблем технологии микроэлектроники и особо чистых материалов Российской академии наук, \\ 142432 Московская область, Черноголовка \\ ฯ E-mail: vdov62@yandex.ru
}

Поступила в Редакцию 23 апреля 2021 г.

В окончательной редакции 28 апреля 2021 г.

Принята к публикации 28 апреля 2021 г.

В результате изучения релаксации фототока в $p-i-n$ GaAs/AlAs-гетероструктурах нами обнаружены резкие особенности, вызванные резонансным туннелированием через электронные уровни квантовых точек в барьерных слоях. Показано, что временны́е интервалы проявления этих резонансов на релаксационных кривых определяются динамикой накопления заряда на дырочных уровнях квантовых точек и рекомбинацией с их участием. Также обнаружены сильные случайные флуктуации фототока в пострезонансной области, обусловленные локальными флуктуациями остаточного заряда на дырочных уровнях квантовых точек. Изучение релаксации в средне- и длинноволновом световом диапазонах подтверждает нашу интерпретацию обнаруженных эффектов.

Ключевые слова: гетероструктуры, фотопропроводимость, квантовые точки.

DOI: $10.21883 /$ FTP.2021.10.51453.9672

\section{1. Введение}

Изучение релаксации фотопроводимости или концентрации (заряда) фотовозбужденных носителей при импульсном световом воздействии уже давно является эффективным инструментом для выяснения механизмов рекомбинации и других, важных для оптоэлектронных, компьютерных и высокочастотных приложений, физических свойств, в разнообразных полупроводниковых и полуметаллических системах. Этот инструмент, например, с успехом применяется в исследованиях объемных полупроводников [1], полупроводниковых гетероструктур [2] и квантовых структур на базе графена [3]. Как уже было продемонстрировано, $p-i-n$ GaAs/AlAs-гетеросистемы со встроенными слоями самоорганизованных квантовых точек (КТ) обладают большим потенциалом для реализации локальных детекторов одиночных фотонов и ячеек оптической памяти нанометрового масштаба [4]. Нами ранее уже обнаружено и изучено проявление на релаксационных кривых $p-i-n \mathrm{GaAs} / \mathrm{AlAs-гетерострук-}$ тур квантовых осцилляций фототока с участием двумерных (2D) уровней треугольной квантовой ямы (ТКЯ) [5]. Однако, как показано далее, данный метод оказывается гораздо более многосторонним и чувствительным к различным другим объектам и процессам в $p-i-n$ гетеросистемах.

В данной работе исследованы зависимости релаксации фототока в $p-i-n \mathrm{GaAs} / \mathrm{AlAs-гетероструктурах} \mathrm{и}$ обнаружены яркие проявления эффектов резонансного туннелирования через „квазилокализованные“ уровни KT в AlAs-барьере, прямой рекомбинации с участием этих уровней и связанной с этим модуляции величины случайных флуктуаций потенциала в ТКЯ, приводящей к подавлению осцилляций.

\section{2. Методика эксперимента и обсуждение результатов}

Исследованные нами образцы были изготовлены на основе $p-i-n \mathrm{GaAs} / \mathrm{AlAs}$ однобарьерных гетероструктур с нелегированными прибарьерными слоями 60 и 100 нм с $p$ - и $n$-областей соответственно, выращенных методом молекулярно-лучевой эпитаксии. В нелегированной $i$-области расположен AlAs 5-нм барьер. В середине барьера встроен слой самоорганизованных InAs квантовых точек. Основные электронные, $E_{Q D}^{e}$, и дырочные, $E_{Q D}^{h}$, энергетические уровни квантовых точек находились на 105 мэВ выше края зоны проводимости и на 15 мэВ ниже края валентной зоны GaAs, т.е. электронное состояние располагалось выше края зоны проводимости GaAs, а дырочное - внутри запрещенной зоны. Верхний $p^{+}$-слой $\mathrm{GaAs}$ толщиной 0.5 мкм был легирован до концентрации $2 \cdot 10^{18} \mathrm{~cm}^{-3}$. Более детально такие структуры описаны в работах [5-7].

Схематическая зонная диаграмма активной области структуры при напряжении $<1.5 \mathrm{~B}$ и слабом освещении показана на рис. $1, a$. Напряжение $V_{b} \approx 1.5 \mathrm{~B}$ является критическим для исследуемых $p-i-n$ GaAs/AlAs-гетероструктур, поскольку отвечает условию плоских зон в них, и интервал $V_{b}>1.5 \mathrm{~B}$ актуален для процессов туннелирования основных носителей, а $V_{b}<1.5 \mathrm{~B}-$ для фотопроводимости и обсуждаемых здесь осцилляций этой величины. Изменение $V_{b}$, начиная с $\sim 1.5 \mathrm{~B}$, в 


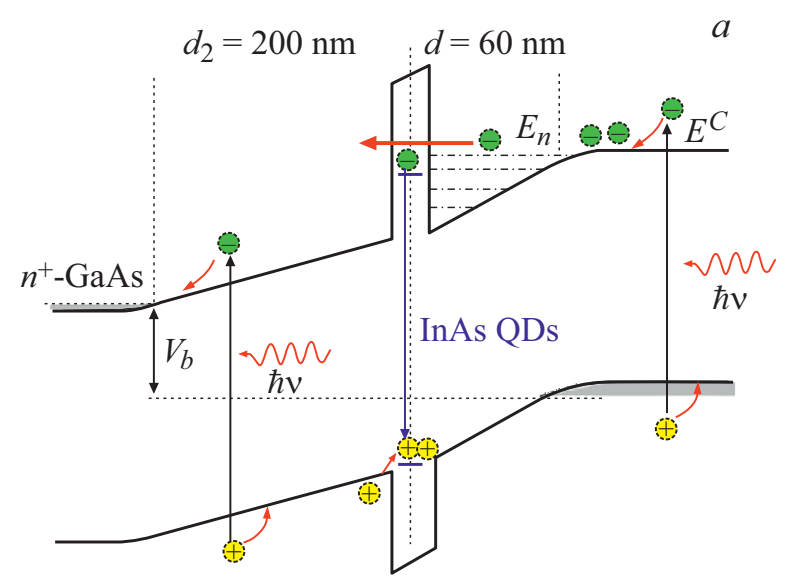

$b$

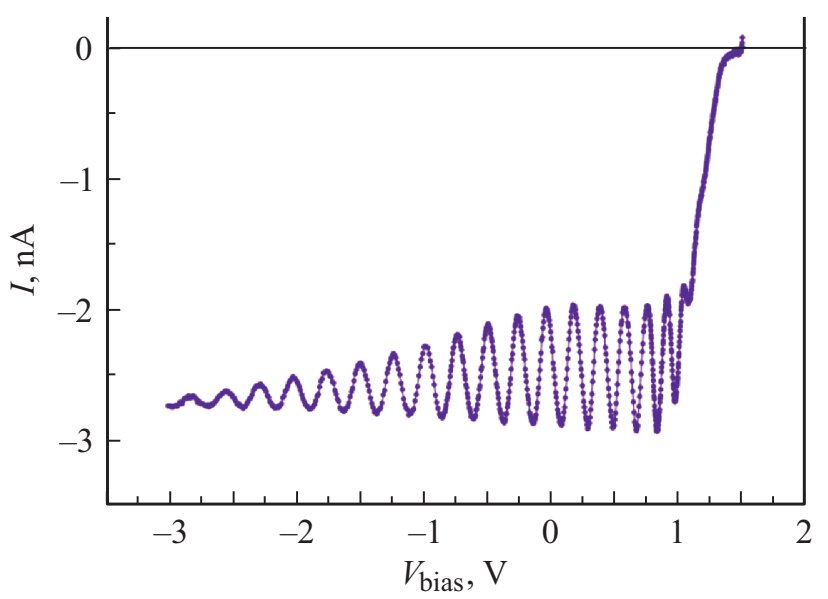

Pис. 1. $a-$ схематическая зонная диаграмма зонной структуры с указанием основных процессов генерации-рекомбинации (вертикальные стрелки) и резонансного туннелирования (красная горизонтальная стрелка). $b$ - осцилляции фототока на обратной ветви ВАХ при $\lambda=405$ нм. (Цветной вариант рисунка представлен в электронной версии статьи).

сторону отрицательных значений приводит к движению электронных уровней размерного квантования $E_{n}$ в треугольной яме, ограниченной AlAs-барьером и краем зоны проводимости $E_{C}$, вверх по энергии и их последовательному совпадению с $E_{C}$, когда и происходят резкие изменения фототока - квантовые осцилляции (см. рис. 1, b) [5-7]. Их проявление на релаксационных зависимостях при импульсном освещении объяснено в [5] следующим образом. С момента начала светового импульса (включения света) генерируемые в нелегированных $i$-слоях электронно-дырочные пары разделяются электрическим полем на электроны и дырки, которые движутся к границам этого слоя в противоположных направлениях и создают в контактных слоях, ограничивающих $i$-слои, заряды противоположного знака. Иными словами, начинает происходить зарядка обкладок емкости $C i$-слоев. Вклад в фотопроводимость и, следовательно, в заряд на обкладках вносят также электроны и дырки, генерируемые светом в контактах, способные продиффундировать в $i$-слой. В результате общий характер временно́й зависимости релаксации тока фотовозбужденных носителей (не принимая во внимание осциллирующую составляющую), так же как и в традиционных $p-i-n$ фотодиодах [1], должен определяться на низких частотах преимущественно соотношением емкостной и резистивной составляющих импеданса активной $i$-области структуры и внешней измерительной цепи и иметь вид, характерный для переходных процессов, например, в дифференцирующих $R C$-цепях, как и в нашей работе [5]. Принципиальным здесь является факт появления заряда на обкладках $i$-слоя в момент сразу после включения света, который должен был бы монотонно изменяться по величине со временем в течение всего периода воздействия светового импульса, когда производится измерение экспериментальной зависимости релаксации фототока от времени, если бы его поведение определялось только соотношением $R$ и $C$. Заряд $Q$ в свою очередь приводит к появлению напряжения смещения между обкладками и, следовательно, к сдвигу зон. Изменение же этого индуцированного фотоносителями напряжения смещения в процессе релаксации фототока (и, соответственно, заряда $Q$ ) вызывает последовательное движение уровней $E_{n}$ в треугольной квантовой яме и, следовательно, модуляцию заряда и фототока на границе квантовой ямы с контактным p-слоем, порождая осциллирующую компоненту экспериментальных временны́х зависимостей. Таким образом, механизм формирования осцилляций вольт-амперной характеристики (BAX) и временны́х зависимостей одинаков, отличие состоит только во внешнем понуждающем параметре. Важно отметить, что в завершение активной фазы процесса релаксации фототока наступает длительный период его неизменности, обусловленный установлением динамического равновесия процессов генерации-рекомбинации носителей во всех слоях структуры и характеризуемый постоянными величинами равновесного заряда на обкладках $Q_{e q u i}$ и равновесного падения напряжения $V_{\text {equi }}$.

Омические контакты изготавливались путем последовательного напыления слоев $\mathrm{AuGe} / \mathrm{Ni} / \mathrm{Au}$ и отжига при $T=400^{\circ} \mathrm{C}$. Для создания оптических мезаструктур диаметром 25-200 мкм применялась стандартная технология химического травления. ВАХ были измерены при уровне шума $<50$ фА. Измерения проводились при температуре $4.2-77 \mathrm{~K}$. В качестве источников светового излучения были использованы светодиоды с длиной волны 650, 525, 405 и 395 нм, импульсные измерения фототока проводились с помощью цифрового осциллографа Tektronix DPO2014.

На рис. 2 представлена временна́я зависимость фототока образца при импульсном освещении светодиодом с длиной волны $\lambda=405$ нм, при напряжении на светодиоде $V_{\text {Led }}=3.125 \mathrm{~B}$ и продолжительности импульса $1 \mathrm{c}$, стационарная ВАХ при постоянном освещении такой интенсивности показана на рис. $1, b$. Как было показано в [5], максимальные значения фототока на переднем и 


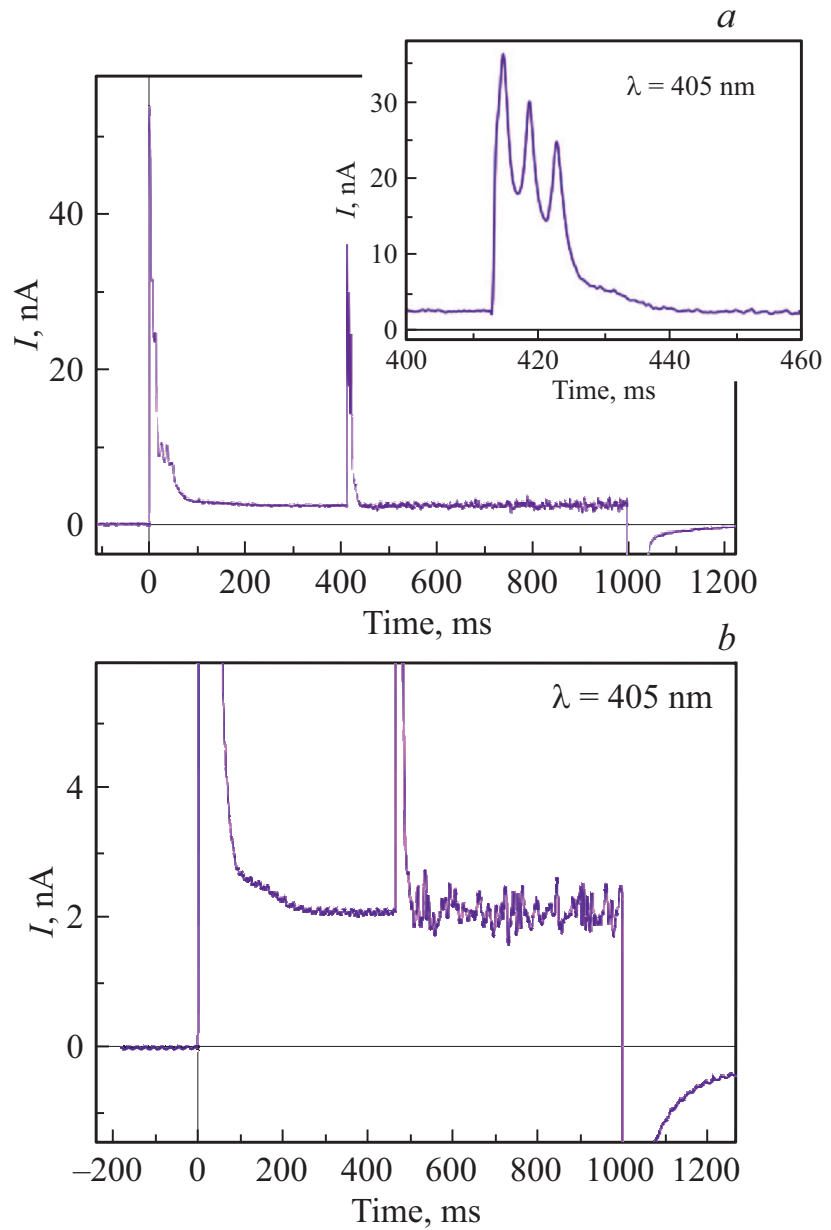

Рис. 2. $a-$ зависимость фототока от времени при импульсном освещении с $\lambda=405$ нм. На вставке подробно показан туннельный резонанс через электронные уровни квантовых точек. $b-$ та же кривая, увеличенная по масштабу тока в области флуктуаций.

заднем фронтах зависимостей и стационарные величины фототока оказываются пропорциональны величине мощности внешнего светового излучения, а на спадах видны осцилляции с периодом обратно пропорциональным мощности. Такой резкий спад фототока (и напряжения на обкладках $i$-слоя) на начальном участке релаксационной зависимости, сопровождающийся квантовыми осцилляциями с участием 2D уровней в ТКЯ, уже объяснен нами ранее [5], и при малых значениях мощности излучения экспериментальные кривые не отличались от наблюдавшихся в этой работе. С увеличением мощности на равновесном участке релаксационных кривых, где величина фототока практически неизменна, как видно из рис. 2 , совершенно непредсказуемо появляется резкая особенность (резкий всплеск фототока), обусловленный проявлением резонансного туннелирования фотовозбужденных электронов из $p$-слоя через электронные уровни КТ в барьере, как будет подробно пояснено далее. Этот всплеск фототока имеет характерную и воспроизводи- мую форму, представляя собой три близко лежащих узких пика тока. Появление этой резонансной особенности может быть объяснено в рамках вышеописанной модели релаксационной зависимости фототока следующим образом. Несмотря на неизменность величины фототока и $V_{\text {equi }}$ на участке динамического равновесия релаксационой кривой, в этот период времени происходит, как уже было показано нами ранее $[7,8]$, относительно медленное накопление положительного заряда дырок, генерируемых в $i$-слое слева от барьера и движущихся к нему под действием электрического поля, на дырочных уровнях КТ в барьере $E_{Q D}^{h}$, приводящее к перераспределению (перелому) линейного падения напряжения $V_{\text {equi }}$ между левым (где падение напряжения уменьшается) и правым (где оно растет) $i$-слоями возле барьера с КТ и смещению/движению электронных уровней КТ, $E_{Q D}^{e}$, вниз по энергии относительно дна зоны проводимости $E_{C}$ в $p$-слое, которые в режиме ,Плоских зон“ находятся на $\sim 115 \mathrm{MB}$ выше $E_{C}$. При совпадении электронных уровней $E_{Q D}^{e}$ с $E_{C}$ происходит резкое резонансное усиление туннелирования через барьер фотовозбужденных электронов, дрейфующих из $p$-слоя с энергией $\sim E_{C}$, при участии $E_{Q D}^{e}$. Наличие трех отдельных пиков на этой резонансной особенности связано с тремя (основным и двумя возбужденными) электронными уровням InAs КТ, наиболее ярко проявляющими себя и в других экспериментах, как, например, в [9]. Открытие канала резонансного туннелирования, кроме того, как сообщалось в $[8]$, поставляя электроны на пустые прежде $E_{Q D}^{e}$, активирует процесс прямой рекомбинации носителей через электронные и дырочные уровни КТ, вызывая резкое уменьшение заряда, накопившегося на $E_{Q D}^{h}$. Это в свою очередь увеличивает деструктивное влияние случайных флуктуаций потенциала в КЯ, индуцированных дырочным зарядом КТ, на возможность экспериментальной регистрации любых особенностей в токе [8], что и проявляется на нашей зависимости в виде случайных скачков фототока со временем во всем интервале после резонанса через $E_{Q D}^{e}$. В данном эксперименте этот эффект проявляется, вероятно, как следствие случайных флуктуаций небольшого заряда, остающегося на $E_{Q D}^{h}$ после активной начальной фазы рекомбинации с $E_{Q D}^{e}$ и установления/достижения равновесия и распределенного вдоль слоя КТ, приводящих к локальным изменениям напряжения в барьере (в области „перелома“ потенциала) и в результате к резким изменениям туннельной прозрачности, проявляющимся в суммарном фототоке.

Этот же механизм роста амплитуды флуктуационного потенциала в плоскости расположения КТ при переходе от состояния с большим аккумулированным на $E_{Q D}^{h}$ зарядом, достаточно равномерно распределенным по плоскости, к малым значениям случайно распределенного заряда на $E_{Q D}^{h}$, проявляющимся в виде так называемого неоднородного уширения в КЯ, приводит к подавлению начальных осцилляций фототока на стационарных ВАХ, как показано на рис. 3 и подробно обсуждено в [8]. 


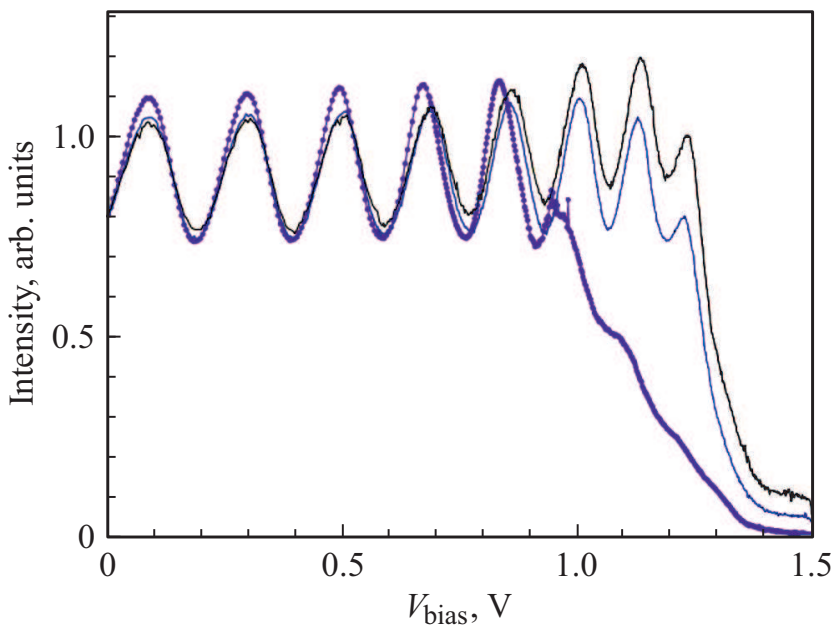

Рис. 3. Подавление начальных осцилляций на стационарных $\mathrm{BAX}$ с ростом мощности освещения при $\lambda=405 \mathrm{HM}$.

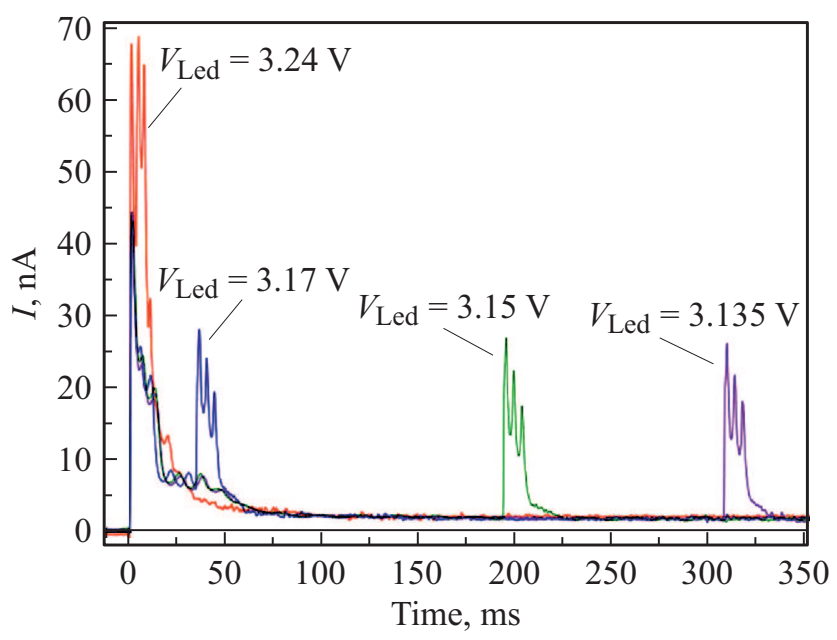

Рис. 4. Зависимости фототока от времени при импульсном освещении с $\lambda=405$ нм для четырех значений мощности освещения.

С увеличением мощности излучения аккумуляция заряда на $E_{Q D}^{h}$ происходит быстрее, что приводит к более раннему „Притягиванию“ уровня $E_{Q D}^{e}$ к $E_{C}$ и отражается в смещении резонанса через него в сторону меньших значений $t$, т. е. ближе к фронту импульса на экспериментальных кривых (см. рис. 4). Уменьшение же мощности приводит к сдвигу этого резонанса в противоположном направлении, вплоть до его исчезновения в случае, когда за время длительности импульса на $E_{Q D}^{h}$ не успевает накопиться заряд, достаточный для втягивания $E_{Q D}^{e}$ под $E_{C}$. Отметим, что появление резонанса $E_{Q D}^{e}$ на импульсных зависимостях через очень большое время (от 50 до $700 \mathrm{мc)}$ после начала импульса с $\lambda=405-395 \mathrm{Hм}$ связано с тем, что количество электронно-дырочных пар, образованных за AlAs-барьером, мало, а большая часть фотонов поглощается в $p^{+}$-слое [8].
Измерения релаксационных зависимостей в средне- и длинноволновом световых диапазонах дали нам независимое подтверждение приведенной выше интерпретации обнаруженных эффектов, поскольку, как показано в [8], с ростом длины волны от фиолетового до красного света происходит последовательное перемещение эффективной глубины поглощения света вдоль основных слоев нашей экспериментальной гетероструктуры, начиная с верхнего p-слоя, приводящее к перераспределению вкладов генерации носителей в этих слоях в суммарный фототок. При облучении образца светодиодами с $\lambda=630$ и 525 нм при примерно тех же величинах стационарного фототока через нашу структуру 1-2 нА проявляются резонансы $E_{Q D}^{e}$ и „флуктационный“ режим фототока (кривые для этих значений длин волн здесь не приводятся из-за их полного подобия с рис. 4 для $\lambda=405$ нм). Кроме того, как и при ультрафиолетовом освещении, начинали подавляться первые осцилляции на соответствующих стационарных ВАХ, как и на рис. 3. Сдвиг резонанса к фронту при приблизительно одинаковой величине стационарного фототока при $\lambda=630$ и 525 нм объясняется тем, что значительно большая доля падающих фотонов сможет проникнуть в $i$-слой слева от барьера, и там будет происходить более эффективная генерация носителей, чем при освещении ультрафиолетом с $\lambda=405 \mathrm{Hм}$, что должно приводить в свою очередь к более быстрому накоплению заряда на $E_{Q D}^{h}$ и, соответственно, к проявлению резонанса через $E_{Q D}^{e}$ при меньших значениях $t$.

\section{3. Заключение}

Таким образом, в результате изучения релаксации фототока в $p-i-n$ гетероструктурах нами обнаружены резкие особенности, вызванные резонансным туннелированием через электронные уровни КТ $E_{Q D}^{e}$, временны́е интервалы проявления которых на релаксационных кривых определяются динамикой накопления заряда на дырочных уровнях КТ $E_{Q D}^{h}$ и рекомбинацией с участием этих уровней, а также сильные случайные флуктуации фототока в пострезонансной области, обусловленные локальными флуктуациями остаточного заряда на $E_{Q D}^{h}$. Изучение релаксации в средне- и длинноволновом диапазонах подтверждает нашу интерпретацию обнаруженных эффектов.

\section{Финансирование работы}

Работа выполнена в рамках государственного задания № 075-00355-21-00.

\section{Благодарности}

Автор выражает благодарность М. Хенини (M. Henini, University of Nottingham, UK ) за изготовление образцов и С.В. Морозову (ИПТМ) за плодотворные обсуждения и техническое содействие. 


\section{Конфликт интересов}

Авторы заявляют, что у них нет конфликта интересов.

\section{Список литературы}

[1] Дж. Гауэр. Оптические системы связи, пер. с англ. (М., Радио и связь, 1989).

[2] В.В. Вайнберг, В.М. Васецкий, Ю.Н. Гуденко, В.Н. Порошин, Н.В. Байдусь, Б.Н. Звонков. ФТП, 46 (12), 1609 (2012).

[3] S. Winnerl, M. Orlita, P. Plochocka, P. Kossacki, M. Potemski, T. Winzer, E. Malic, A. Knorr, M. Sprinkle, C. Berger, W.A. de Heer, H. Schneider, M. Helm. Phys. Rev. Lett., 107, 237401 (2011).

[4] O. Makarovsky, E.E. Vdovin, A. Patane, L. Eaves, M.N. Makhonin, A.I. Tartakovskii, M. Hopkinson. Phys. Rev. Lett., 108, 117402 (2012).

[5] Ю.Н. Ханин, Е.Е. Вдовин. ФТП, 52 (6), 591 (2018).

[6] E. E. Vdovin, M. Ashdown, A. Patane, L. Eaves, R.P. Campion, Yu.N. Khanin, M. Henini, O. Makarovsky. Phys. Rev. B, 89, 205305 (2014).

[7] Ю.Н. Ханин, Е.Е. Вдовин, О. Макаровский, М. Хенини. Письма ЖЭТФ, 102 (11), 830 (2015).

[8] Е.Е. Вдовин, Ю.Н. Ханин. Письма ЖЭТФ, $113(9), 605$ (2021).

[9] A.E. Belyaev, S.A. Vitusevich, L. Eaves, P.C. Main, M. Henini, A. Forster, W. Reetz, S.V. Danylyuk. Nanotechnology, 13, 94 (2002).

Редактор Г.А. Оганесян

\section{Manifestations of resonant tunneling processes and random potential fluctuations with the participation of quantum dot levels in the relaxation of the photocurrent of $p-i-n$ GaAs/AlAs heterostructures}

Yu.N. Khanin, E.E. Vdovin

Institute for Problems of Microelectronics

Technology and High-Purity Materials,

Russian Academy of Sciences,

142432 Moscow Region, Chernogolovka

Abstract As a result of studying the relaxation of the photocurrent in $p-i-n \mathrm{GaAs} / \mathrm{AlAs}$ heterostructures, we discovered/ registered sharp features caused by resonant tunneling through the electronic levels of quantum dots in the barrier layers. It was shown that the time intervals of the manifestation of these resonances on the relaxation curves are determined by the dynamics of charge accumulation at the hole levels of quantum dots and by recombination with their participation.Strong random fluctuations of the photocurrent in the postresonant region, caused by local fluctuations of the residual charge at the hole levels of quantum dots, were also found.The study of relaxation in the medium and long wavelength light ranges confirms our interpretation of the observed effects. 